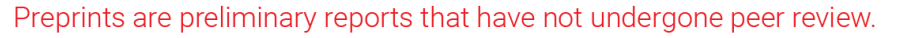 \\ They should not be considered conclusive, used to inform clinical practice, or referenced by the media as validated information. \\ Dynamic Changes of the Colonic Microbiota in Rat during Severe Acute Pancreatitis
}

sizhen Wang

Research Institute of General Surgery

\section{Zhihui Tong}

Research Institute of General Surgery

\section{Weiqin Li}

Research Institute of General Surgery

\section{Qiurong Li}

reseach institution of general surgery

Jieshou Li ( $\nabla$ wsizhen@126.com )

Jinling Hospital, Nanjing

\section{Research article}

Keywords: Severe acute pancreatitis, bacterial translocations, colonic flora dysbiosis

Posted Date: October 27th, 2020

DOI: https://doi.org/10.21203/rs.3.rs-65424/v1

License: (c) (1) This work is licensed under a Creative Commons Attribution 4.0 International License. Read Full License 


\section{Abstract}

Background: The bacterial contamination of pancreatic necrosis in acute pancreatitis is supposed to occur through translocation of intestinal bacteria. To date, the pathophysiology of the underlying bacterial translocation is poorly understood. Increased gut permeability and gut flora dysbiosis may be the initial phenomenon in this process. The present study investigated the colonic microbiota alteration pattern as well as observed colonic epithelium change in rats with SAP.

Method: Denaturing gradient gel electrophoresis (DGGE) was used to monitor the colonic microbiota of control rats and experimental rats that 2, 6,and 12 hours following severe acute pancreatitis which was induced by retrograde injection of sodium taurocholate into biliopancreatic duct. Colonic samples and its contents at $1 \mathrm{~cm}$ distal to the cecal-colonic junction was harvested for morphological studies, streptavidin-peroxidase immunohistochemistry examination.

Results: Denaturing gradient gel electrophoresis of colonic microbiota showed that gut flora pattern changed early after severe acute pancreatitis, differed significantly at 6 hours of model was induced, and then started to recover towards normal pattern. The specific dysbiosis were characterized by Escherichia coli proliferation and Lachnospiraceae and Lactobacilli reduction. As shown by immunohistochemical staining, greatly reduced expressions of ZO-1 protein were detected in rats with SAP. And the structures of junctional complexes were disrupted and the gaps between cell junctions were wider after the SAP induced.

Conclusions: Severe acute pancreatitis results in colonic flora dysbiosis. And tight junction in rats with SAP was disrupted in the intestinal epithelium. Both of them may be a key factor contributing to bacterial translocations in rats with SAP.

\section{Background}

Vertebrates harbor a densely populated resident microbial community, which consists of bacteria, viruses and fungi, particularly in mucosal organs, such as the oral cavity and the intestine[1]. The intestinal tract is one of the most complex and diverse ecosystems, comprising up to 1014 cells of 300-500 different microbial species, many of which have never been cultured. In healthy individuals, Gram-negative Proteobacteria and Bacteroidetes, and Gram-positive Firmicutes, such as Clostridiales and Lactobacillales, are the major phyla among intestinal eubacteria, whereas methanogens are the predominant intestinal archaea[2]. Although the gut microbiota has previously been studied in the context of inflammatory diseases, it has recently become clear that this microbial community has a beneficial role during normal homeostasis, modulating the host's immune system as well as influencing host development and physiology, including organ development and morphogenesis, and host metabolism[3].

Severe acute pancreatitis (SAP) is a potentially lethal disease with considerable morbidity and mortality. It is often accompanied by systemic infammatory response syndrome, sepsis, and even organ dysfunction $[4,5]$. It has become apparent that there are two phases of acute pancreatitis: an early phase 
(usually within the first week of onset) and a subsequent phase occurring after the first week of onset of the disease. The first phase is characterized more by the presence or absence of organ failure. The late phase is characterized by infectious complications following bacterial translocation of intestinal bacteria and late mortality. And the infection of pancreatic necrosis by gut bacteria is a major cause of morbidity and mortality in patients with severe acute pancreatitis. Two important factors which have been proposed as promoting bacterial translocation in patients with severe acute pancreatitis are physical injury of the gastrointestinal epithelium and the gut flora dysbiosis.

Intestinal microbial community changes have been investigated in critical ill patients and animal models using conventional bacteriological analysis techniques, while few study applies culture-independent methods [6]. In our previously study have provided a detailed description on the prevalence of bacteremia and characteristic of bacterial species in patients with acute pancreatitis. We also have demonstrated an association between the bacteremia and the disease severity, which enabled us to better understand a potential role of bacterial translocation in the pathogenesis of septic complication in acute pancreatitis [7].

In this study, using denaturing gradient gel electrophoresis (DGGE) method, we aimed to investigate intestinal microbiota alteration pattern and identifying specific bacterial species that differ significantly as well as observing intestinal epithelium change in rat models of severe acute pancreatitis.

\section{Methods}

\section{Animals and Surgical Procedure}

Male Sprague-Dawley rats (200-250g) (Laboratory Animals Center, Jinling Hospital) were housed in a controlled environment ( $12 \mathrm{hr}$ daylight cycles) in groups of two rats per cage, and were allowed free access to standard chow and water. The experiments were carried out under the supervision of Jinling Hospital Animal Care and Use Committee. Twenty-two rats were randomized into four groups of 6 animals each.

SAP model was created essentially according to the previously described procedure[8]. Briefly, All rats underwent intraperitoneal injection of ketamine $(100 \mathrm{mg} / \mathrm{kg})$ as anesthesia. $3.5 \%$ sodium taurocholate (dissolved in normal saline) was injected into pancreatic and bile duct retrogradely with micro-pump set at a speed of $0.1 \mathrm{ml} / \mathrm{min}$ and volume of $1 \mathrm{ml} / \mathrm{kg}$ body weight. The main pancreatic and bile duct was clamped just below the liver. This clamp remained in place throughout the intraductal infusion to prevent misdirected flow into the biliary system. The abdomen was then closed. For the sham operation group, the abdomen was opened and the pancreatic and bile duct were flipped and $1 \mathrm{ml} / \mathrm{kg}$ body weight normal saline was injected pancreatic and bile duct retrogradely before closing abdomen. A group of six rats were sacrificed 2,6 , and $12 \mathrm{~h}$ after operation to collect samples. All procedures and sample collections were under sterile techniques. Blood samples and tissues were harvested for further analysis. All samples were stored at $-80^{\circ}$ for further analysis. Colonic samples and its contents at $1 \mathrm{~cm}$ distal to the cecal- 
colonic junction were collected. All operations were performed under anesthesia. Rats were then killed by over-dosed anesthesia (pentobarbital :250 mg/kg).

\section{DGGE of intestinal Microbiota}

Luminal bacterial DNA was extracted from $200 \mathrm{mg}$ of intestinal contents, using the QIAamp DNA Stool Mini Kit (QIAGEN, Hilden, Germany), according to the manufacturer's instructions, and stored at $-20^{\circ} \mathrm{C}$ until use. The hypervariable V3 region of the $16 \mathrm{~S}$ ribosomal RNA gene was amplified by polymerase chain reaction (PCR) using forward primer (GC357f 5'-

CGCCCGGGGCGCGCCCCGGGCGGGGCGGGGGCACGGGGGGATTACCGCGGCTGCTGG-3') and reverse primer (518r 5'-CCTACGGGAGGCAGCAG-3'). The $50 \mu \mathrm{l}$ PCR reaction mixture were: $1 \mu \mathrm{l}$ of extracted bacterial DNA, $5 \mu$ l of $10 \times$ PCR buffer, $1 \mu \mathrm{l}$ of dNTP mixture (2.5 mM each), $1 \mu$ l of each primer (10 pM), $0.5 \mu \mathrm{l}$ of Taq-Polymerase $(5 \mathrm{U} / \mu \mathrm{l}), 40.5 \mu \mathrm{l}$ sterile water. PCR was run in a 2720

thermal cycler (Applied Biosystems, Foster City, CA) as a touchdown PCR. The program was $94^{\circ} \mathrm{C}(5$ min), followed by 20 cycles of $94^{\circ} \mathrm{C}$ for $(30 \mathrm{sec}), 65^{\circ} \mathrm{C}(30 \mathrm{sec})$ decreased by $0.5^{\circ} \mathrm{C}$ for each cycle, and 68 ${ }^{\circ} \mathrm{C}(30 \mathrm{sec})$, additional $10 \mathrm{cycles}$ of $94^{\circ} \mathrm{C}(30 \mathrm{sec}), 55^{\circ} \mathrm{C}(30 \mathrm{sec})$, and $68^{\circ} \mathrm{C}(30 \mathrm{sec})$, and a final extension at $68^{\circ} \mathrm{C}(7 \mathrm{~min})$.

DGGE gels were prepared using a DCode Universal Mutation Detection System (BIO-RAD, Hercules, CA) with $8 \%(\mathrm{w} / \mathrm{v})$ polyacrylamide (acrylamide: Bis 37.5:1) in $1 \times$ TAE $(20 \mathrm{mM}$ Tris, $10 \mathrm{mM}$ acetate, $0.5 \mathrm{M}$ EDTA, pH 7.4) in denaturing gradients ranging from 35 to $50 \%$. While $100 \%$ chemical denaturant solution consists of $7 \mathrm{M}$ urea and $40 \%$ formamide. At both outer lanes of each gel, a common sample was loaded for gel normalization to allow comparison between gels. Electrophoresis was run in $1 \times \mathrm{TAE}$ at $60^{\circ} \mathrm{C}$, first at $200 \mathrm{~V}$ for 10 minutes and then at $120 \mathrm{~V}$ for 7.5 hours. All gels were stained with SYBR Green I (Invitrogen) for $30 \mathrm{~min}$, and viewed under U.V illumination. Images were captured by ChemiDoc XRS Camera (BIO-RAD, Hercules, CA).

\section{Analysis of DGGE Profile}

Gel images were aligned using Adobe Photoshop CS4 by running common samples on both outer sides of each gel, to allow comparison of two gels in one profile. Digitizing the profile was done by Quantity One software (version 4.6). Individual bands in each sample lane were marked automatically by the software, followed by manual correction if necessary. Bands occupying the same position in different lanes were matched and identified as the same band type, using the match tolerance of $0.5 \%$. The relative quantity of a given band was expressed as a fraction (\%) of the sum of all defined bands in the same lane. The quantitative information derived from relative band quantities per band type per sample was exported as a data matrix. The method for calculating similarity between two lanes was the Dice Coefficient. Unweighted pair group method with arithmetic mean was used to compare the similarity of samples in a dendrogram. Principal component analysis (PCA) was performed using Canoco (version 4.5) based on the data matrix mentioned above. Species richness was calculated as the total number of 
individual lane's bands. Shannon's evenness index, Shannon's diversity index were generated on quantitative information.

\section{Sequencing of DGGE Bands}

Bands of interest from DGGE gels were cut out with a sterile scalpel, placed into a single Eppendorf tube, and incubated in $20 \mu \mathrm{l}$ of sterile water overnight at $4^{\circ} \mathrm{C}$. Four microliters of the overnight solution was used as template for PCR re-amplification. Using the forward primer (without GC clamp) (357f 5'ATTACCGCGGCTGCTGG -3') and the reverse primer (518r 5'-CCTACGGGAGGCAGCAG-3'), the $50 \mu \mathrm{l}$ PCR reaction mixture were: $4 \mu \mathrm{l}$ of template DNA, $5 \mu$ l of $10 \times$ PCR buffer, $1 \mu \mathrm{l}$ of dNTP mixture (2.5 mM each), $1 \mu \mathrm{l}$ of each primer $(10 \mathrm{pM}), 0.5 \mu \mathrm{l}$ of Taq-Polymerase $(5 \mathrm{U} / \mu \mathrm{l}), 37.5 \mu \mathrm{l}$ sterile water. The touchdown program was $94^{\circ} \mathrm{C}(5 \mathrm{~min})$, followed by 20 cycles of $94^{\circ} \mathrm{C}$ for $(30 \mathrm{sec}), 65^{\circ} \mathrm{C}(30 \mathrm{sec})$ decreased by $0.5^{\circ} \mathrm{C}$ for each cycle, and $68^{\circ} \mathrm{C}(30 \mathrm{sec})$, additional 15 cycles of $94^{\circ} \mathrm{C}(30 \mathrm{sec}), 55^{\circ} \mathrm{C}(30 \mathrm{sec})$, and $68^{\circ} \mathrm{C}(30$ $\mathrm{sec})$, and a final extension at $68^{\circ} \mathrm{C}(7 \mathrm{~min})$. After purification, $\mathrm{PCR}$ products were sequenced using the Sanger's method on a 3730 DNA Analyzer (Applied Biosystems, Foster City, CA). The obtained sequences were compared with NCBI GenBank databases using the BLAST tool. The phylogenetic tree was constructed using the MEGA 4.0 program in the method of neighbor-joining based on evolutionary distances.

\section{Real-time PCR}

Bacterial species that characterize the predominant dysbiosis in rat model of severe acute pancreatitis injury derived from the DGGE comparative analyses were quantified by quantitative PCR using the 7300 Real-Time PCR System (Applied Biosystems, USA). The primer pairs for the bacteria were reported in previous studies (see Table, Supplemental Digital Content 1). The real-time PCR was carried out in a $20 \mu \mathrm{l}$ total reaction mixture: $1 \mu \mathrm{l}$ of template DNA, $0.4 \mu \mathrm{l}$ of each primer, $10 \mu \mathrm{l} 2 \times$ SYBR Green Realtime PCR Master Mix (TOYOBO, Japan) and $8.2 \mu \mathrm{l}$ of sterile water. The amplification program consisted of one cycle at $95^{\circ} \mathrm{C}$ for $5 \mathrm{~min} ; 40$ cycles at $95^{\circ} \mathrm{C}$ for $15 \mathrm{sec}, 55^{\circ} \mathrm{C}$ for $60 \mathrm{sec}$. Specific bacteria $16 \mathrm{~S}$ rRNA gene amount was normalized to total bacteria $16 \mathrm{~S}$ rRNA gene amount using the $2^{-\Delta C t}$ method. Quantification values are represented by fold changes relative to control rats.

\section{Histopathology}

Colonic samples was fixed in $10 \%$ formalin. Paraffin sections were prepared and stained with hematoxylin and eosion. The degree of colonic tissue injury was evaluated by a pathologist in a blinded set-up using a grading system described previously by Chiu et al. (14) The scales were: grade 0, no damage; grade 1, subepithelial space development at the apex of the villus; grade 2, subepithelial space extension; grade 3, massive lifting of epithelia, including a few denuded tips; grade 4, denuded lamina propria with cellularity increase; grade 5 , lamina propria disintegration and hemorrhage. 


\section{Immunofluorescence}

One centimeter segments of colon was harvested, washed with normal saline (0.9\%), snapped frozen in liquid nitrogen and stored at $-80^{\circ} \mathrm{C}$ until use. Tissues were embedded in OCT compound and cut into 5 $\mu \mathrm{m}$ frozen sections, followed by fixation in acetone at $-20{ }^{\circ} \mathrm{C}$ for 5 minutes. After 15 minutes of blocking with $1 \%$ bovine serum albumin, sections were incubated with polyclonal anti-occludin $(1: 150)$ or polyclonal anti-ZO-1 (1:150) (Zymed Laboratories Inc, San Francisco, CA) as primary antibodies overnight at $4{ }^{\circ} \mathrm{C}$. Sections were probed with Alexa Fluor 488-conjugated anti-rabbit secondary antibodies (1:150) (Invitrogen) for 30 minutes at $4{ }^{\circ} \mathrm{C}$, followed by counterstained with DAPI (1:500) (Invitrogen). Images were obtained with Leica TCS SP5 confocal scanning microscope (Leica Microsystems, Heidelberg $\mathrm{GmbH}$, Mannheim, Germany).

\section{Immunohistochemistry}

Immunohistochemical staining was performed on formalin-fixed, paraffin-embedded specimens. A standard immunohistochemical technique was applied. After blocking endogenous peroxides and proteins, specimens were exposed to primary antibody. Rabbit polyclonal anti-zonula occludens $\triangle \mathrm{ZOQ}-1$ antibodies were purchased from Zymed Laboratories Inc (San Francisco,CA). Horseradish peroxidaseconjugated secondary antibody was purchased from Santa Cruz Biotechnology Inc (Santa Cruz, CA).

\section{Statistical Analyses}

Data are presented as mean \pm standard error of the mean. Statistical analysis was performed using oneway analysis of variance followed by post hoc Bonferroni's multiple comparison test. For non-parametric data, Kruskal-Wallis followed by Dunn's multiple comparison test was used. A $p$ value of less than .05 was considered significant. The analyses were conducted using GraphPad Prism version 5.

\section{Results}

\section{Feasibility of surgical procedure}

3.5\% sodium taurocholate was injected into pancreatic and bile duct retrogradely were successfully achieved in all animals in an average of $(15 \pm 2)$ minutes per animal when the procedure was mastered. The cumulative death rate of 8 hours in the SAP group was about $50 \%$, and the rate was increased to $75 \%$ on 12 hours, while no death happened in the control group.

\section{Rat autopsy}


Autopsy of dead rats were made. When the abdomen was opened, much yellow turbid or bloody exudates with slight fume would come out from the peritoneal cavity. The intestine expanded about 2-3 times with gas and effusion in it. The pancreas was swollen and dark-colored with some local hemorrhagic spots. Peripancreatic omentum and retroperitoneum were always found with some yellow-white saponification spots. Peripancreatic organs such as the small intestine, colon, stomach, spleen and part of the liver were always seen adhered together as a mass.

\section{Pancreatic Histopathology.}

Under light microscopy, partial necrosis of the pancreas, glandular destruction, inflammatory cell infiltrates, hemorrhage and swelling, and edema of nonnecrotic glands were observed. Microscopic pancreatic damage was pronounced in pancreatitis animals in the first 12 hours and showed a progressive disease course with pancreatic hemorrhage, leukocyte infiltration at first and interstitial edema, acinar cell necrosis, later (Fig. 1B-1D). The control animals were observed without such abnormities, with normal pancreatic gland lobules and cells (Fig. 1A).

\section{Dynamic Changes of the Colonic Microbiota}

The colonic bacterial DNA in rats with severe AP was evaluated using 16S rDNA-based technique. DGGE profiles of proximal colonic flora from rats at different hours after SAP induction showed shifts of colonic microbial community composition (Fig. 2). It could be observed that some bands became more intense and appeared more prevalently, such as band C14. As shown in Fig. 3, banding pattern similarities were measured using Dice coefficient and UPGMA as a cluster method. There were two primary clusters. The above cluster including control and 2 hours group The down cluster contained all 6-h samples and samples of 2 hours after model induced. The dendrogram demonstrated that the community profiles of rats of 6,12-h groups and control group were clearly distinct (53\% between clusters). There also nearly $60 \%$ distinct between control and 2-h group except for only two individuals.

Principal component analysis (PCA) of DGGE fingerprints of colonic microbiota (Fig. 4) confirmed and exhibited the temporal changes of colonic flora according to different time points more thoroughly. The distance between 2 data points represents the extent of difference between of the 2 rats' gut microbial compositions. Microbial structure of 6 and 12 hours of reperfusion showed a separation from normal colonal microbiota by PCA axis 1 and 2. Furthermore, 12 hours group colonic microbiota differed considerably from normal samples by moving towards right of PCA axis 1 and up of PCA axis 2. And 6-h group moved toward right of PCA axis 1. Different from 6-h and 12-h experimental groups, the 2-h-group colonic flora profiles do not segregated from the control groups.

We examined colonic content community diversity based on the DGGE profiles. Rats that in 6 hours and 12 hours groups had a slightly higher diversity of colonic bacteria compared to control and 2 hours groups, whereas there was no significant difference(Fig. 5A).The species richness was lower in the 6 
hours group versus the other three time points groups. However, no statistically significant changes were observed about the species richness(Fig. 5B). There was no significant difference in the colonic microbial evenness for all groups of rats in the experiments(Fig. 5C).

These bands were excised from the DGGE gel and sequenced to identify which species they are. The bands were assigned to a bacterial species based on the highest (Q90\%) sequence identity with GenBank sequences through BLAST analysis (Table 1).In general, the microbial shifts occurred in two major patterns: bacterial groups that increased after SAP model was induced, including Prevotella melaninogenica (C14), Enterococcus hirae (C15), Escherichia coli(C16), Desulfotomaculum acetoxidans (C17), Clostridium lentocellum(C10), Acidovorax avenae subsp(C11), Desulfotomaculum reducens(C21), Lactobacillus gasseri(C2) and Eubacterium cellulosolvens(C26); four bacterial groups that increased after SAP model was induced, including Lactobacillus amylovorus(C7), Prevotella denticola(C25), Micavibrio aeruginosavorus(C20) and Clostridium saccharobutylicum(C13).

Table 1

The results of identities of bands cut from DGGE gels.

\begin{tabular}{|llll|}
\hline Bands & Closest relatives & Accession No. & Tendency \\
\hline C14 & Prevotella melaninogenica & NC_014371.1 & Increase \\
\hline C15 & Enterococcus hirae & NC_018081.1 & Increase \\
\hline C16 & Escherichia coli & NC_018658.1 & Increase \\
\hline C17 & Desulfotomaculum acetoxidans & NC_013216.1 & Increase \\
\hline C10 & Clostridium lentocellum & NC_015275.1 & Increase \\
\hline C11 & Acidovorax avenae subsp. avenae & NC_015138.1 & Increase \\
\hline C21 & Desulfotomaculum reducens & NC_009253.1 & Increase \\
\hline C2 & Lactobacillus gasseri & NC_008530.1 & Increase \\
\hline C26 & Eubacterium cellulosolvens & NZ_CM001487.1 & Increase \\
\hline C7 & Lactobacillus amylovorus & NC_014724.1 & Decrease \\
\hline C25 & Prevotella denticola & NC_015311.1 & Decrease \\
\hline C20 & Micavibrio aeruginosavorus & NC_016026.1 & Decrease \\
\hline C13 & Clostridium saccharobutylicum & NC_022571.1 & Decrease \\
\hline
\end{tabular}

Quantitative real-time PCR was performed to verify the changes found by DGGE. Escherichia coli16S rRNA gene content was significantly higher from 6 to $12 \mathrm{~h}$ after SAP model was induced (Fig. 6A) $(\mathrm{P}<$ 0.05). Whereas numbers of Lactobacilli decreased significantly $(P<0.05)($ Fig. 6, B)

Villous edema, exfoliation, deformity, lodging, shortening, mucosal erosion, and infiltration of inflammatory cells were all observed in the colon of the 3 groups. Following SAP model induced, 
disintegration of the gut epithelial lining and deformity could be observed at $2 \mathrm{~h}$ group (Fig. 7B). 12 hours after SAP induced showed the highest injury scores compared with the control group (Fig. 7E).

Transmission electron microscopic analysis provided details to the pathological changes of SAP rat colonic structure. The colonic epithelia of rats in sham group had well organized microvillus layer and tight-junction structure (Fig. 8a). On the other hand, the colonic epithelia of SAP rats almost completely lost epithelial structure and microvilli with destructed organelle and tight-junctions (Fig. 8b).

Immunohistochemistry for TJ protein ZO-1 stainings were continuously and uniformly distributed at the apical part of epithelial cells in Groups A. Some weak staining was also seen in the cytoplasm, lateral part of epithelial cells and subnucleus. In the Groups B $\triangle$ C and D, the continuity of ZO-1 was disrupted ,discontinuous and vague (Fig. 9).

\section{Discussion}

The clinical outcome of patients presenting with SAP was significantly associated with bacteria crossing the intestinal barrier and then invading organ systems, resulting in super infections associated with pancreatic necrosis(9). Bacterial translocation has been hypothesized to occur during SAP since this is often accompanied by bacteriemia or endotoxiemia even without sepsis. Although the pathogenesis of intestinal bacterial translocation associated with SAP has yet to be elucidated, several mechanisms have been proposed, including: i) Altered permeability of the intestinal mucosa, ii) a disruption of the indigenous gut flora, and iii) decreased host defenses (12). In the present study, acute pancreatitis induced by retrograde intraductal injection of $3.5 \%$ sodium taurocholate, a model with about $75 \% 12$-hour mortality, was used. Its severity is progressive over time, can be easily modulated, and its morphology closely resembles that of human pancreatitis. We also have showed observations of two pathophysiology progresses in rat model of SAP. First, we characterized the colonic flora change pattern and identified species whose amounts differed significantly after the model induced. Second, we showed that colonic epithelial barrier impairment.

The route and factors of bacterial translocation from the gut in those with acute pancreatitis has not been clarified and may depend on the underlying pathogenetic cause. And the relationship between AP and the mucosal changes seen in this rat model is not completely understood. The major mechanism that has been postulated for increased intestinal permeability is splanchnic hypoperfusion and ischemia. The cause of splanchnic hypoperfusion is mainly due to hypovolemia(13). It is possible that severe volume depletion accompanying AP might cause a partial ischemic injury to the intestinal mucosa. In our previously study we showed that colonic epithelial barrier impairment during rats intestinal IschemiaReperfusion Injury (10). In fact, alterations derived from gut ischemia reportedly can induce BT (4). Moreover, the systemic release of proinflammatory mediators such as interleukin 1, tumor necrosis factor, and histamine could induce the morphologic changes seen and result in increased permeability of the mucosa. Our study showed that villous edema, exfoliation, deformity, lodging, shortening, mucosal erosion, and infiltration of inflammatory cells in the colon of the experimental groups and the colonic 
epithelia of SAP rats almost completely lost epithelial structure and microvilli with destructed organelle and tight-junctions under the transmission electron microscopic analysis.

Intestinal motility, which also is altered during AP, results in overgrowth of indigenous flora in the mecum and ileum. This was confirmed in our model and, by itself, can induce BT (11). In early stage of SAP often accompanied with severe volume depletion and insufficient resuscitation. Our previously study showed that gut ischemia-reperfusion affected the colonic microbiota pattern dynamically and promoted overgrowth of Escherichia coli and Prevotella oralis. Proliferation of Lactobacilliin the later reperfusion phase was concordant with the colonic epithelium recovery time course (10).

The TJ proteins found in epithelium are critical for the maintenance of mucosal integrity and resistance to bacterial invasion. SAP can damage the integrity and function of intestinal mucosa by infuencing the expression of TJ proteins [4]. TJ proteins, such as ZO-1, claudins and occludin, are indispensable to maintain the function of TJs. This study was undertaken to investigate whether TJ protein (ZO-1) disruption occurs in the colonic mucosa in rat model of SAP. ZO-1 was significantly decreased. In the sham operation group, the staining for ZO-1 was mainly located at the apical part of epithelial cells, displaying a continuous and uniform distribution with weak cytoplasmic staining. In the Groups of SAP rats, ZO-1 staining appeared discontinuous and vague.

\section{Conclusion}

In summary, the translocation of gut bacteria and gut derived endotoxin due to intestinal barrier dysfunction induced by SAP may be the aggravating cause of SAP. Our study may provide an experimental basis for underlying mechanisms of BT during SAP.

\section{Abbreviations}

DGGE:denaturing gradientgel electrophoresis; PCA:principal component analysis;SAP severe acute pancreatitis; NCBI:national center for biotechnology information;BLAST:basic local alignment search tool;TEMED:N,N,N',N'-Tetramethylethylenediamine; OCT: optimum cutting temperature embedding medium; DNA:Deoxyribonucleic acid; SIRS: Systemic inflammatory response syndrome; ICC: interstitial cells of Cajal; ZO-1 :zonula occludens-1; PCR: polymerase chain reaction; TJ: tight junction; BT: Bacterial translocation; DAPI: 4',6-diamidino-2-phenylindole; RNA: Ribonucleic Acid

\section{Declarations}

\section{Ethics approval and consent to participate:}

The experimental protocol was established, according to the ethical guidelines of the Helsinki Declaration and was approved by the Ethics Committee of Jinling Hospital. The experiments were carried out under the supervision of Jinling Hospital Animal Care and Use Committee. 


\section{Consent for publication:}

Not applicable

\section{Availability of data and material:}

Not applicable

\section{Competing Interest:}

The authors declare no conflict of interest.

\section{Authors' contributions:}

SZW, WQL, QRL and JSL developed the idea of the study, participated in its design and coordination and helped to draft the manuscript. SZW and ZHT contributed to the acquisition and interpretation of data. JSL provided critical review and substantially revised the manuscript. All authors read and approved the final manuscript.

\section{Acknowledgements:}

Not applicable

\section{References}

1. Nobuhiko Kamada, Grace Y Chen, Naohiro Inohara, Gabriel Núñez. Control of pathogens and pathobionts by the gut microbiota. Nature Immunology. 2013, 14,685-690

2. Dridi, , Raoult, D, Drancourt, M. Archaea as emerging organisms in complex human microbiomes. Anaerobe.2011;17:56-63.

3. Felix Sommer, and Fredrik Bäckhed. The gut microbiota - masters of host development and physiology. Nat Rev Microbiol. 2013;11:227-38

4. Huang L, Jiang Y, Sun Z, Gao Z, Wang J, Zhang D. Autophagy Strengthens Intestinal Mucosal Barrier by Attenuating Oxidative Stress in Severe Acute Pancreatitis. Dig Dis Sci. 2018;63:910-919.

5. Barlass U, Dutta R, Cheema H, George J, Sareen A, Dixit A, Yuan Z, Giri B, Meng J, Banerjee S, Banerjee S. Morphine worsens the severity and prevents pancreatic regeneration in mouse models of acute pancreatitis.Gut.2018;67:600-602.

6. Hayakawa M, Asahara T, Henzan N, Murakami H, Yamamoto H. Dramatic Changes of the Gut Flora Immediately After Severe and Sudden Insults. Dig Dis Sci. 2011, 56: 2361-2365. 
7. Li Q, Wang C, Tang C, He Q, Li N, Li J. Bacteremia in the Patients With Acute Pancreatitis as Revealed by 16S Ribosomal RNA Gene-Based Techniques. Crit Care Med. 2013;41:1938-50

8. Schmidt J, Rattner DW, Lewandrowski K, Compton CC, Mandavilli U, Knoefel WT, Warshaw AL. A Better Model of Acute Pancreatitis for Evaluating Therapy. Ann Surg. 1992; 215:44-56

9. Lichtman SM: Bacterial translocation in humans. J Pediatr Gastroenterol Nutr .2001;33: 1-

10. Wang F, Li Q, Wang C, et al. Dynamic alteration of the colonic microbiota in intestinal ischemiareperfusion injury [J]. PLoS One. 2012;7:e42027.

11. Nieuwenhuijs VB, Verheem A, van Duijvenbode-Beumer H, Visser MR, Verhoef J, Gooszen HG, Akkermans LM: The role of interdigestive small bowel motility in the regulation of gut microflora, bacterial overgrowth, and bacterial translocation in rats. Ann Surg.1998;228:188-193

12. Xuecheng Sun, Yingying Shao, Yin Jin, et al. Melatonin reduces bacterial translocation by preventing damage to the intestinal mucosa in an experimental severe acute pancreatitis rat model. Experimental and Therapeutic Medicine, 2013. 6: 1343-1349

13. Manik Sharma,V ikas Sachdev, Namrata Singh, et al. Alterations in intestinal permeability and endotoxemia in severe acute pancreatitis. T ropical Gastroenterology 2012;33:45-50.

14. Chiu CJ, McArdle AH, Brown R, Scott HJ, Gurd FN. Intestinal mucosal lesion in low-flow states. I. A morphological, hemodynamic, and metabolic reappraisal. Arch Surg. 970;101:478-83.

\section{Figures}



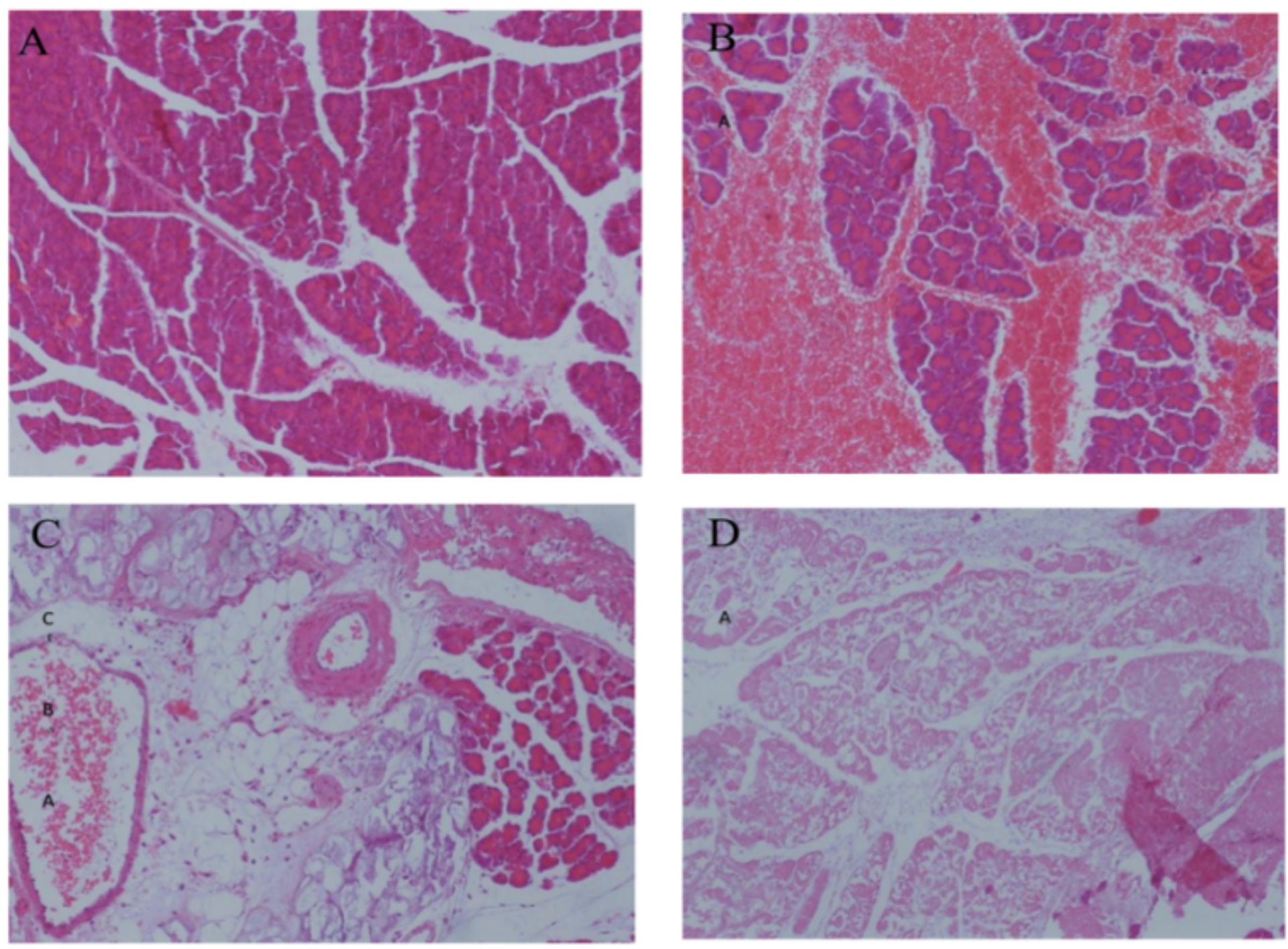

\section{Figure 1}

Morphological changes of the pancreas. A. Control animal: The glandular architecture was entirely normal. B. 2hours: The histopathologic appearance of pronounced edema, marked interstitial leukocyte infiltration, intrapancreatic hemorrhage. C. 6hours: Moderate to severe edema, moderate cell necrosis are the principal features. D.12hours: The glandular architecture was entirely necrosis. 


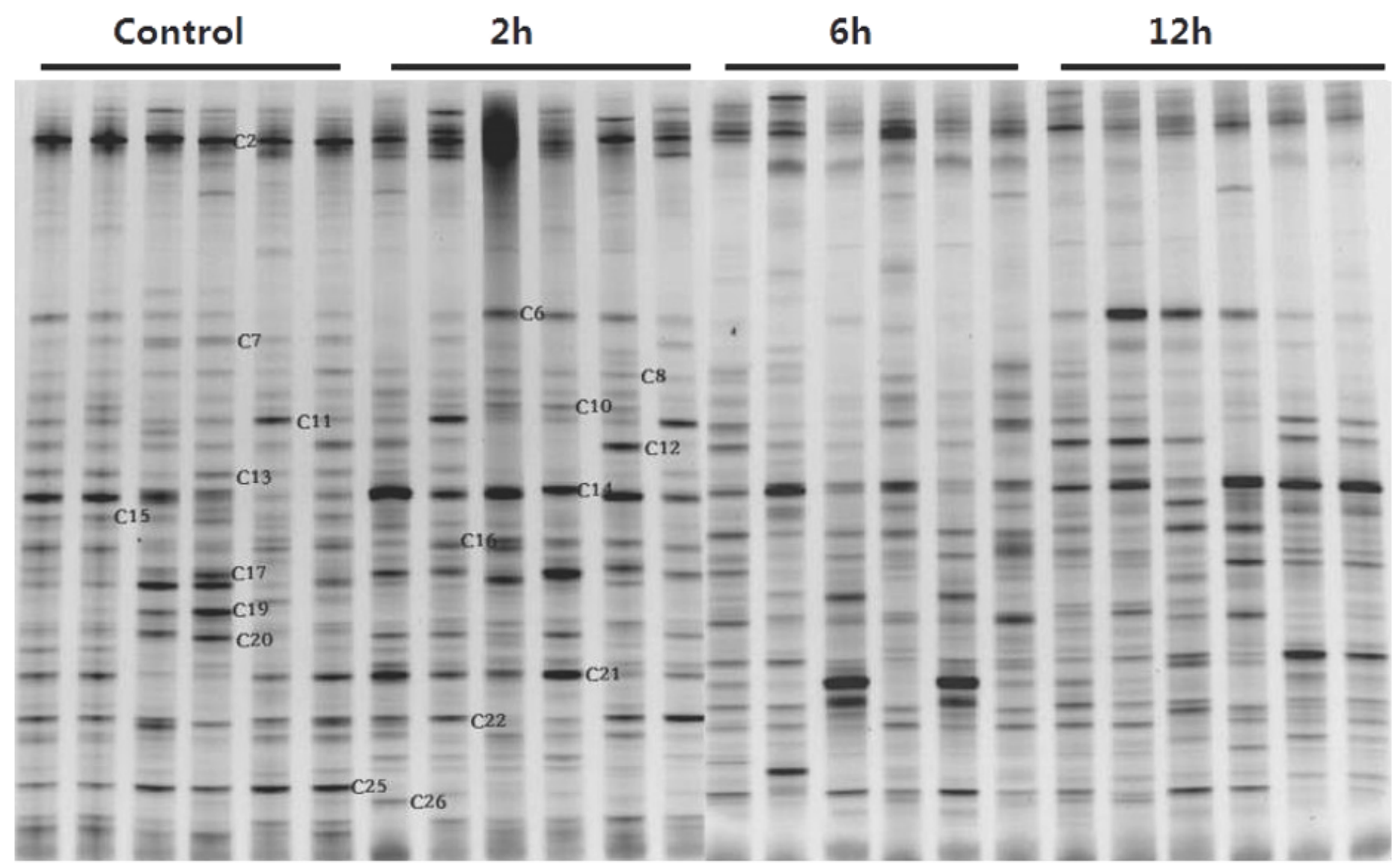

Figure 2

Severe acute pancreatitis changes the colonic microbiota dynamically shown in DGGE profiles. Sample numbers above lanes indicate hours after SAP model induced. Each band represents a bacterial clone. Band numbers and arrowheads indicate the position of bands excised for sequence analyses. (e.g. "C2" means band $\mathrm{C} 2$ ). 


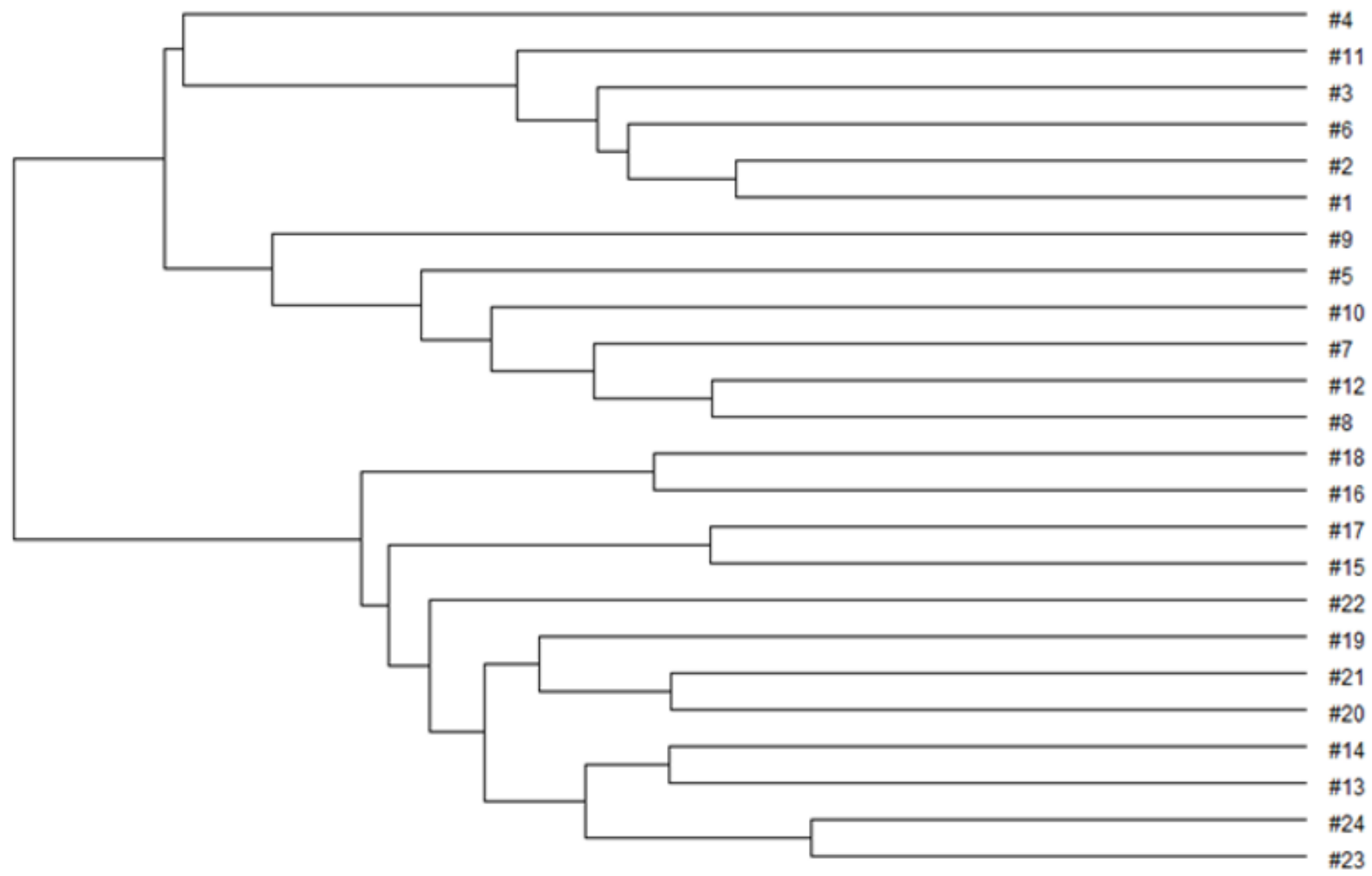

\section{Figure 3}

Dendrogram of DGGE profiles using UPGMA. Metric scale above the figure denotes degree of similarity. The numbers denote samples in a group at the particular hours in rats after SAP induced. Control group: \#1-\#6; 2 hours: \#7-\#12; 6 hours: \#13-\#18; 12hours: \#19-\#24. 


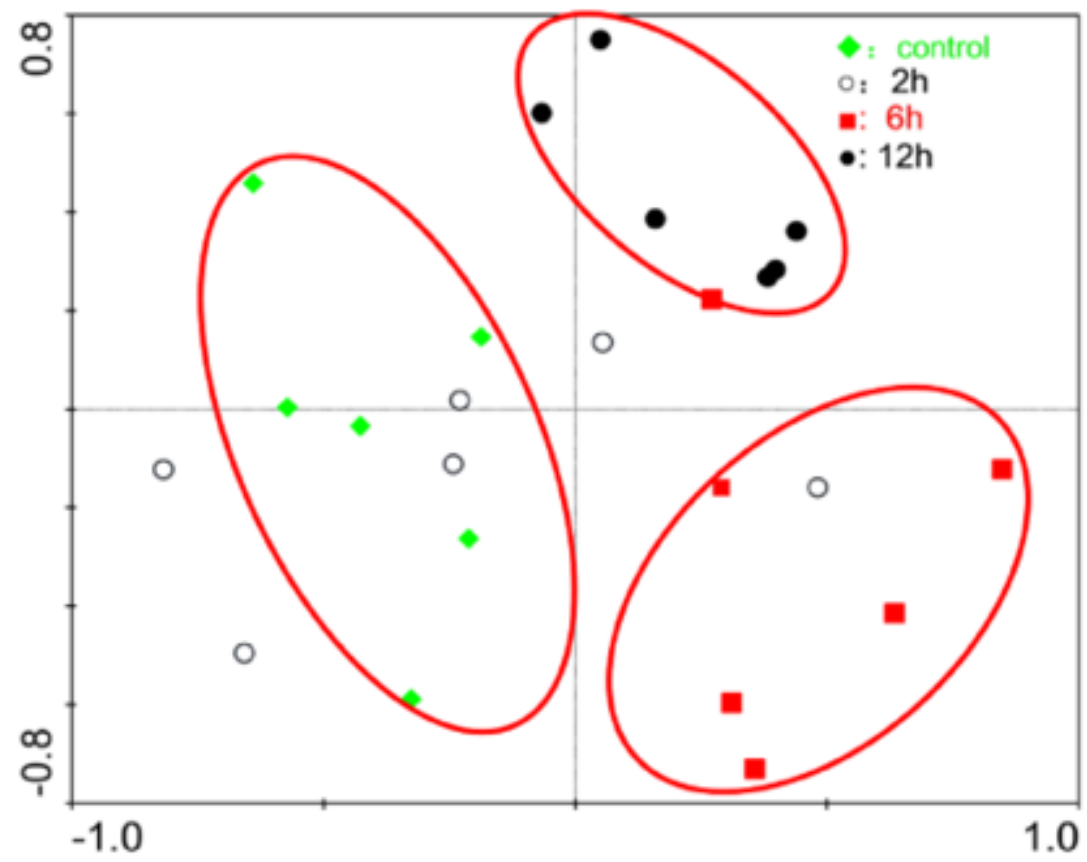

Figure 4

Principal component analysis (PCA) of colonic microbiota based on DGGE fingerprinting. Each color symbol represents a single sample according to model induced time.

A

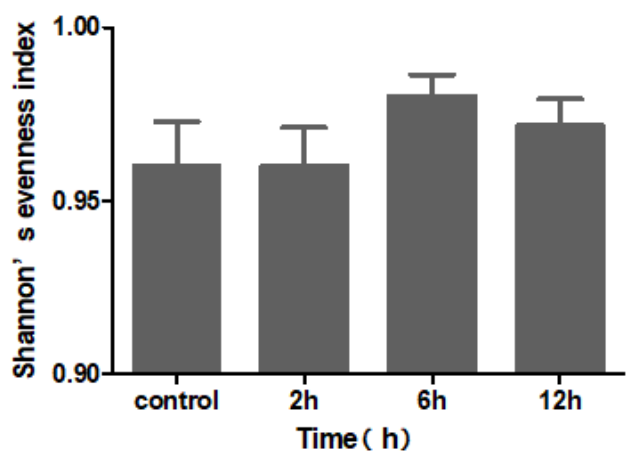

B

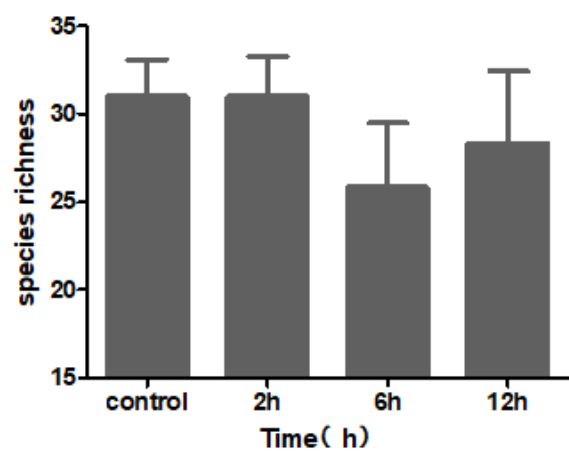

C

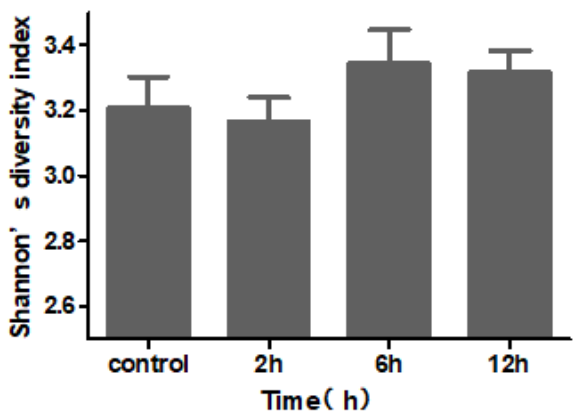

Figure 5

Colonic microbiota diversity comparison. A, Shannon's diversity index. B, Species richness. C, Shannon's evenness index. 
A

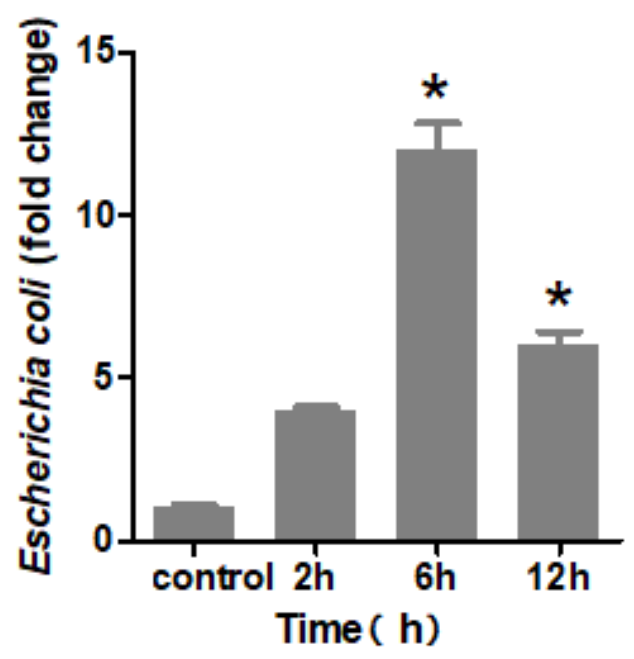

B

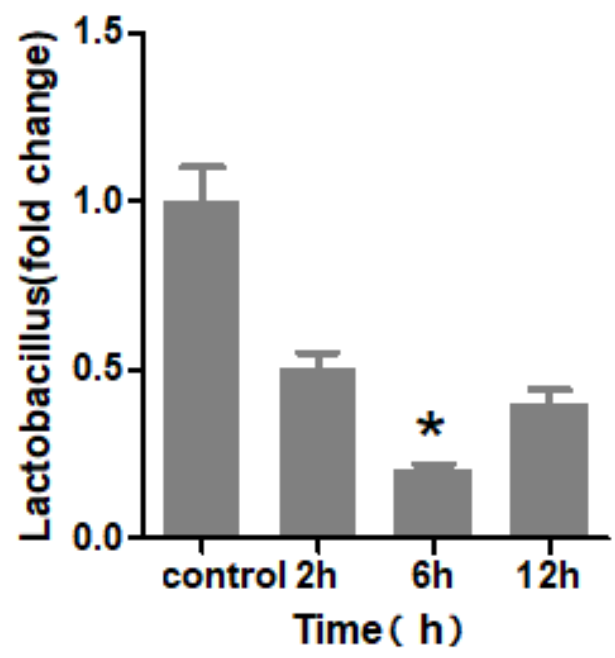

Figure 6

Quantitative analysis of characteristic bacterial species. The relative quantity of specific groups of bacteria was determined by real-time PCR of $16 S$ rRNA gene of (A)E. coli and (B) Lactobacilli, and normalized to total bacteria $16 \mathrm{~S}$ rRNA gene amount using the 2- $\triangle \mathrm{CTmethod.} \mathrm{Quantification} \mathrm{values} \mathrm{are}$ represented by fold changes relative to control rats. ${ }^{*}<0.05$ between group vs. control group.
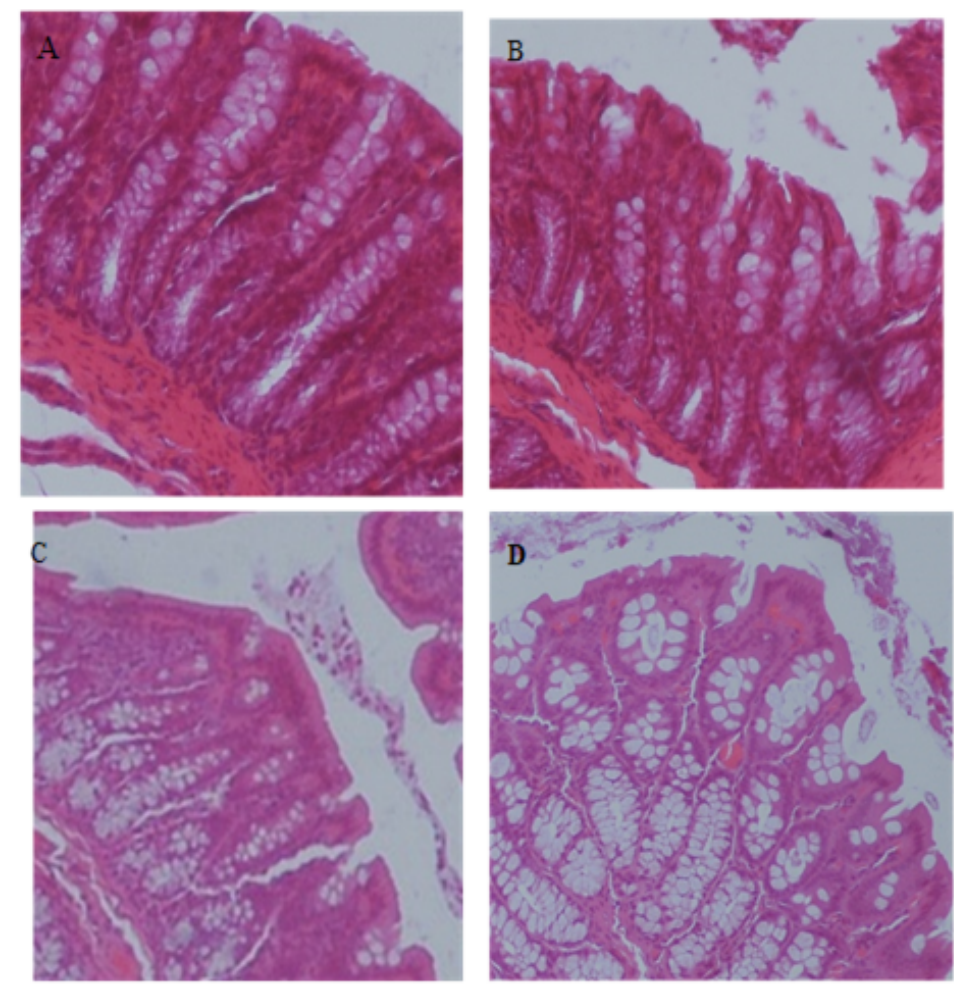

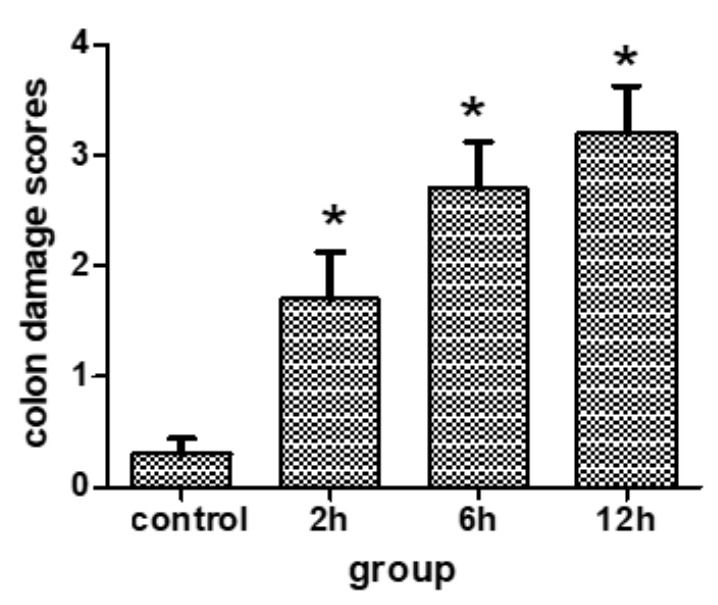

Figure 7 
Dynamic changes of colonic epithelial histopathology. (A-D), representative graphs of control, 2-hr,6-hr and 12-hr after SAP model was induced, respectively. (E), comparison of colon damage scores of control group with the grades of different model time.
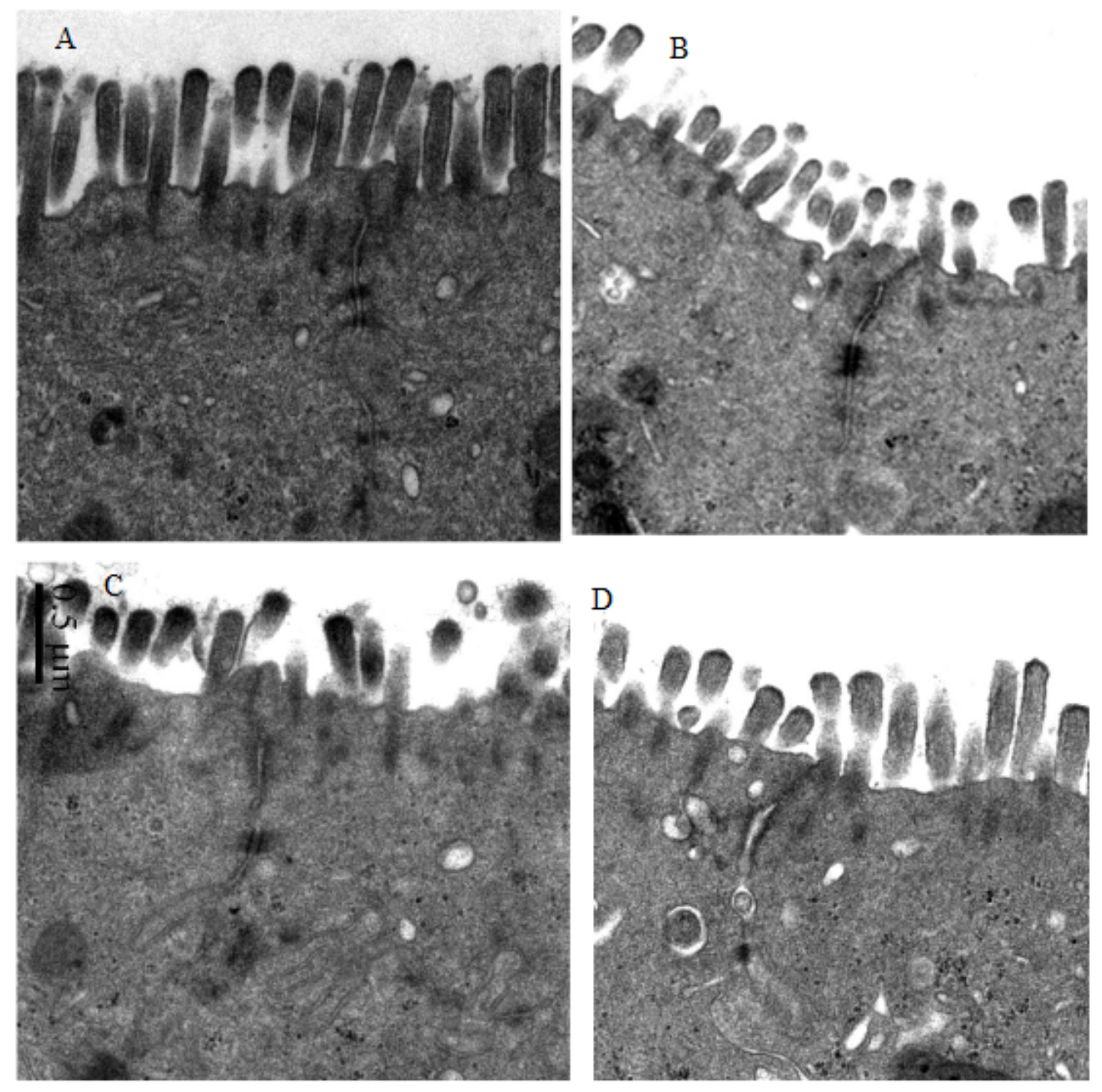

\section{Figure 8}

Colonic mucosa electron microscopic changes $(910,000)$ A: Control mucosa showing long and tight microvilli and intact tight junction (arrowhead); B-D: Shortened microvilli with disintegration of tight junction (arrowhead) of colonic mucosa at different model time. 

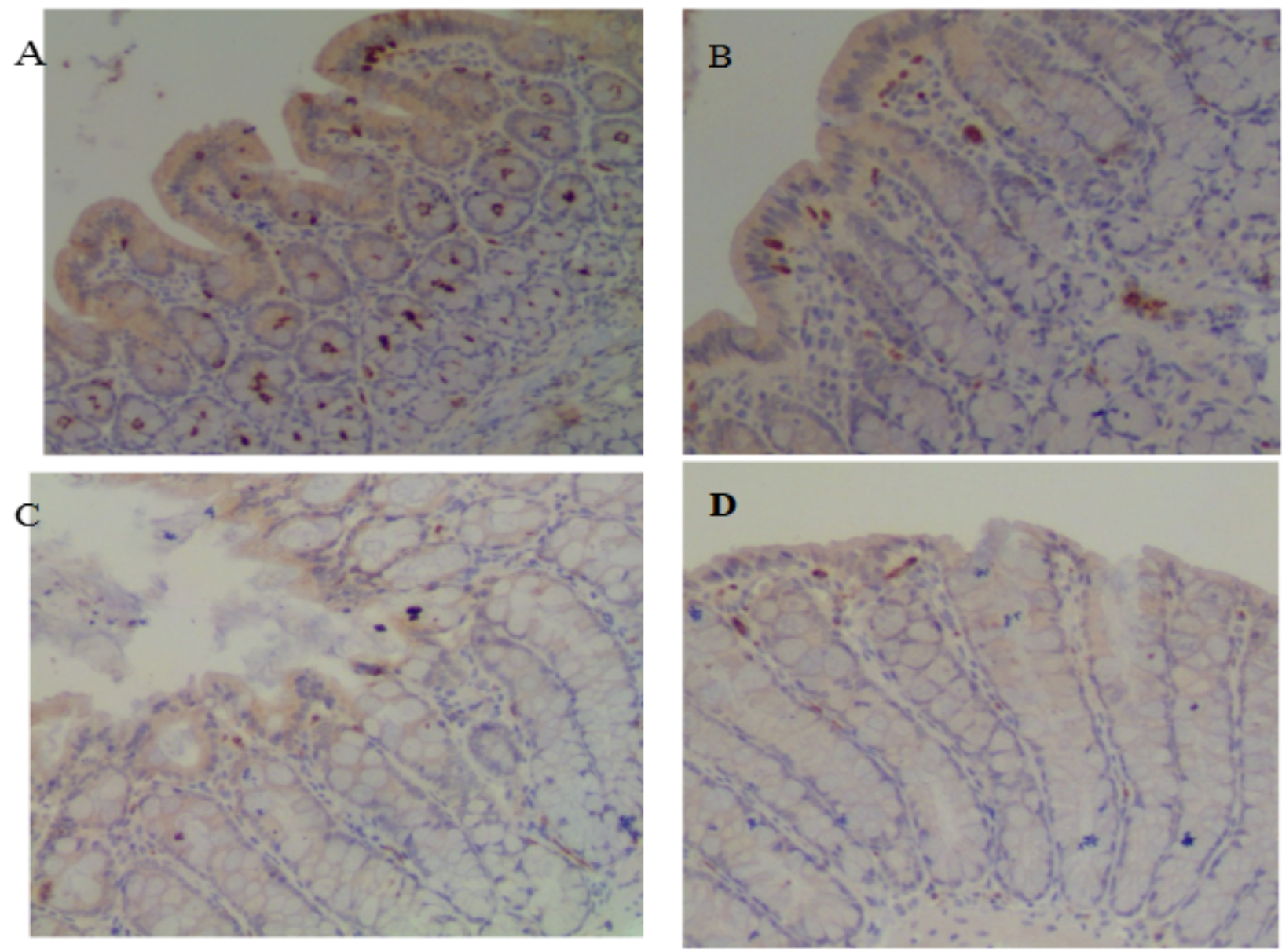

\section{Figure 9}

Immunohistochemical expressions of tight junction protein $₫ Z O-1 \rrbracket$ in the colonic epithelium . In Group A, the staining for ZO-1 was mainly located at the apical part of epithelial cells, displaying a continuous and uniform distribution with weak cytoplasmic staining. In the Groups B $\square$ C and D, ZO-1 staining appeared discontinuous and vague.

\section{Supplementary Files}

This is a list of supplementary files associated with this preprint. Click to download.

- AuthorChecklistE10only.pdf

- supplement2.docx 